\section{Nivel educativo y su asociación con niveles de actividad física en Chile}

\author{
ÁLEX GARRIDO-MÉNDEZ ${ }^{1, a *}$, CARLOS MATUS-CASTILLO ${ }^{1, a *}$, \\ FELIPE POBLETE-VALDERRAMA ${ }^{2,11, b}$, \\ CAROL FLORES-RIVERA ${ }^{3, \mathrm{~d}}$, FANNY PETERMANN-ROCHA ${ }^{4,5, \mathrm{c}}$, \\ FERNANDO RODRÍGUEZ-RODRÍGUEZ ${ }^{6, a}$, \\ JAIME VÁSQUEZ-GÓMEZ ${ }^{7, a}$, XIMENA DÍAZ-MARTÍNEZ ${ }^{8, \mathrm{~b}}$, \\ ANA ROSA BELTRÁN ${ }^{9, e}$, CARLOS CELIS-MORALES ${ }^{5,10,12, a}$ \\ en representación del Grupo de Investigación ELHOC.
}

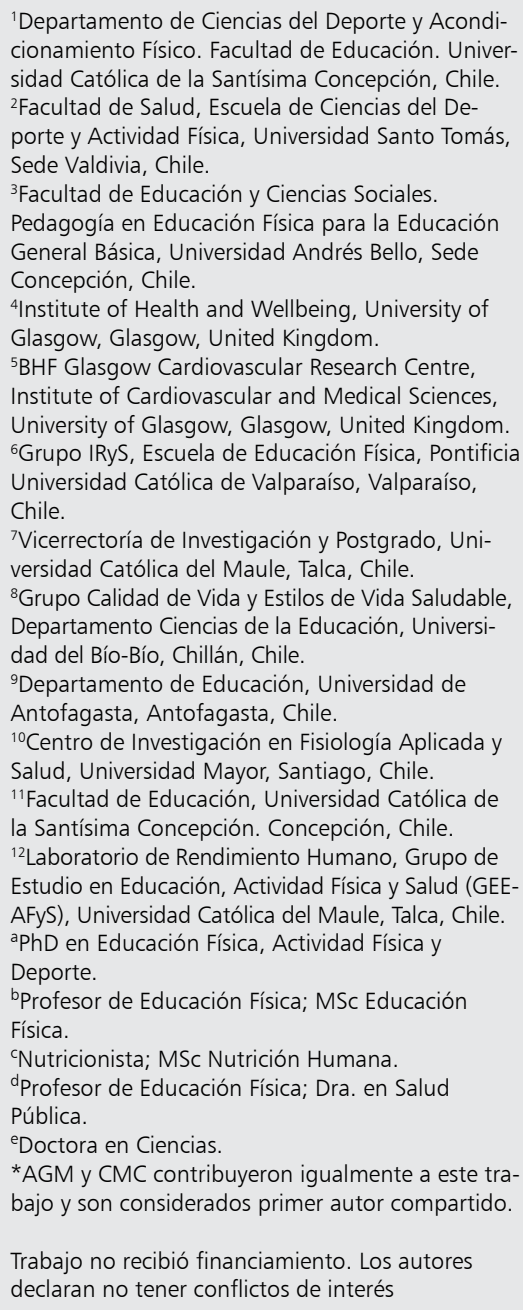
cionamiento Físico. Facultad de Educación. Universidad Católica de la Santísima Concepción, Chile. ${ }^{2}$ Facultad de Salud, Escuela de Ciencias del Deporte y Actividad Física, Universidad Santo Tomás, Sede Valdivia, Chile

${ }^{3}$ Facultad de Educación y Ciencias Sociales.

Pedagogía en Educación Física para la Educación General Básica, Universidad Andrés Bello, Sede Concepción, Chile.

${ }^{4}$ Institute of Health and Wellbeing, University of Glasgow, Glasgow, United Kingdom.

${ }^{5}$ BHF Glasgow Cardiovascular Research Centre, Institute of Cardiovascular and Medical Sciences, University of Glasgow, Glasgow, United Kingdom. ${ }^{6}$ Grupo IRyS, Escuela de Educación Física, Pontificia Universidad Católica de Valparaíso, Valparaíso, Chile.

${ }^{7}$ Vicerrectoría de Investigación y Postgrado, Universidad Católica del Maule, Talca, Chile. ${ }^{8}$ Grupo Calidad de Vida y Estilos de Vida Saludable, Departamento Ciencias de la Educación, Universidad del Bío-Bío, Chillán, Chile.

'Departamento de Educación, Universidad de Antofagasta, Antofagasta, Chile.

${ }^{10} \mathrm{Centro}$ de Investigación en Fisiología Aplicada y Salud, Universidad Mayor, Santiago, Chile.

"Facultad de Educación, Universidad Católica de la Santísima Concepción. Concepción, Chile. ${ }^{12}$ Laboratorio de Rendimiento Humano, Grupo de Estudio en Educación, Actividad Física y Salud (GEEAFyS), Universidad Católica del Maule, Talca, Chile. aphD en Educación Física, Actividad Física y Deporte.

brofesor de Educación Física; MSc Educación Física.

'Nutricionista; MSc Nutrición Humana.

dProfesor de Educación Física; Dra. en Salud Pública.

eDoctora en Ciencias.

*AGM y CMC contribuyeron igualmente a este trabajo y son considerados primer autor compartido.

Trabajo no recibió financiamiento. Los autores declaran no tener conflictos de interés

Recibido el 28 de marzo de 2019, aceptado el 9 de marzo de 2020.

\section{Correspondencia:}

Dr. Carlos Celis-Morales

BHF Glasgow Cardiovascular Research Centre 126 University Avenue. Glasgow University. Glasgow, United Kingdom.

G12 8TA

carlos.celis@glasgow.ac.uk

(Rev Med Chile 2020; 148: 295-303)
Key words: Education; Physical Activity; Sex.

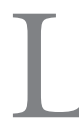

a inactividad física es reconocida como un importante factor de riesgo en el desarrollo de enfermedades crónicas no transmisibles $(\mathrm{ECNT})^{1-6}$. Bajos niveles de actividad física (AF) se asocian a un mayor riesgo de enfermedades cardiovasculares, hipertensión, diabetes, síndrome metabólico y cáncer ${ }^{1-6}$. Si bien los beneficios asociados a la salud de la práctica regular de AF son conocidos, cerca de un $27,5 \%$ de la población adulta a nivel mundial es físicamente inactiva, es decir, realiza menos de 150 minutos de AF de intensidad moderada a vigorosa $o<75$ minutos de intensidad vigorosa por semana ${ }^{7}$.

En Chile, los resultados de la última encuesta 
nacional de salud señalan que un $35,1 \%$ de los chilenos son físicamente inactivos ${ }^{8}$. En este contexto, cabe preguntarse, ¿qué factores podrían influir en los niveles de AF de la población chilena? Se ha demostrado que factores sociodemográficos se asocian a importantes diferencias en los niveles de AF en población adulta ${ }^{9-11}$, destacando el nivel socioeconómico, el sexo, la edad, el estado civil, así como también el nivel educacional ${ }^{9-11}$. Sin embargo, estudios recientes han indicado que personas con un nivel bajo de escolaridad presentan una menor adherencia a conductas saludables y estilos de vida saludable, lo cual aumentaría el riesgo de desarrollar enfermedades cardiovasculares y el riesgo de mortalidad prematura en personas con bajos niveles de escolaridad o nivel socio-economico ${ }^{12}$. Teniendo en cuenta estos antecedentes, el objetivo de este estudio fue investigar el nivel de adherencia a las recomendaciones de AF según los años de estudio en población chilena.

\section{Material y Métodos}

\section{Diseño de la encuesta}

La población correspondió a todos los participantes de la Encuesta Nacional de Salud de Chile 2016-2017 (ENS 2016-17) ${ }^{8}$. De esta se incluyeron a 6.174 personas (2.297 hombres y 3.877 mujeres) de 15 años y más que tenían información disponible sobre AF y años de estudio. La ENS 2016-2017 es una encuesta de prevalencia, realizada en hogares, con una muestra nacional, probabilística, estratificada y multietápica, con representatividad nacional, regional, y de área urbana/rural. Al expandir la muestra a población nacional mediante la aplicación de los factores de expansión sugeridos por la ENS 2016-17 ${ }^{8}$ esta representaría a 14.518.969 de chilenos (7.131.326 hombres y 7.387.643 mujeres). El protocolo de la ENS 2016-17 fue aprobado por el Comité de Ética de la Escuela de Medicina de la Pontificia Universidad Católica de Chile, y todos los participantes firmaron un consentimiento informado ${ }^{8}$.

\section{Clasificación de los años de estudio}

Los años de estudio cursados fueron recolectados mediante cuestionario ${ }^{8}$. Los participantes reportaron entre 0 a 22 años de escolaridad; sin embargo, para el objetivo de este estudio se clasificó a la población en cinco categorías según sus años de estudio $(<4,5-8,9-12,13-16$ y $>16$ años). Otras variables sociodemográficas como edad y sexo también fueron recolectadas mediante cuestionarios ${ }^{8}$.

\section{Variables antropométricas}

Estas mediciones fueron realizadas por personal capacitado y han sido descritas en extenso en otra publicación ${ }^{8}$. El peso corporal y estatura fueron utilizados para determinar el índice de masa corporal (IMC: $\mathrm{kg} / \mathrm{m}^{2}$ ). El estado nutricional se clasificó de acuerdo a las recomendaciones de la OMS (bajo peso: $<18,5 \mathrm{~kg} / \mathrm{m}^{2}$; normal: 18,5 a 24,9 $\mathrm{kg} / \mathrm{m}^{2}$; sobrepeso: $\geq 25,0 \mathrm{a}<29,9 \mathrm{~kg} / \mathrm{m}^{2}$; obesidad: $\left.\geq 30,0 \mathrm{~kg} / \mathrm{m}^{2}\right)^{8}$. El perímetro de cintura (PC) fue medido en la línea axilar media, en el punto medio entre el reborde costal y la cresta iliaca, con una cinta métrica en centímetros. La obesidad central fue definida como un $\mathrm{PC} \geq 88 \mathrm{~cm}$ para mujeres y $\geq 102 \mathrm{~cm}$ para hombres ${ }^{8}$.

\section{Medición de los niveles de actividad física}

Los niveles de AF de la población fueron determinados con el cuestionario "Global Physical Questionnaire" (GPAQ v2) ${ }^{13}$, el cual ha sido validado internacionalmente y también en población latina ${ }^{14-16}$. Este instrumento midió el tiempo destinado a las actividades físicas relacionadas con el transporte (Ej. caminar y andar en bicicleta) y a $\mathrm{AF}$ de intensidad moderada y vigorosa realizadas en el tiempo libre y en el trabajo. Cada una de estas actividades fue expresada en minutos por día. Para determinar los niveles de AF de intensidad moderada a vigorosa (MVPA, de la definición en Ingles moderate-to-vigorous Physical activity) y los niveles de AF total, las variables fueron expresadas en METs (metabolic-energy-equivalents), que es una unidad utilizada frecuentemente para estimar la intensidad de distintos tipos de $\mathrm{AF}^{13}$. Se considera que $1 \mathrm{MET}$ es equivalente al gasto energético basal de una persona en reposo (equivalente a $1 \mathrm{kcal} / \mathrm{kg} / \mathrm{h})^{13}$. Se consideró como punto de corte para inactividad física un gasto energético $<600$ METs/min/semana según las recomendaciones de la OMS y especificaciones de la guía de análisis de $\mathrm{GPAQ}^{13}$. El nivel de sedentarismo fue determinado mediante auto-reporte de tiempo (minutos por día) destinado a actividades que involucren estar sentado o reclinado durante el tiempo libre o de trabajo $^{13}$. 


\section{Análisis estadísticos}

Los análisis fueron realizados con STATA 15 MP. El comando "svy" fue utilizado para muestras complejas y los análisis fueron ponderados a población nacional mediante la utilización de los factores de expansión proporcionados por la ENS 2016-17 .

Las características de la población según las categorías de años de escolaridad $(<4,5-8,9-12$, 13-16 y $>16$ años) fueron ponderados para población nacional y presentados como promedio para variables continuas y como prevalencia para variables categóricas con sus respectivos intervalos de confianza del 95\% (95\% IC).

Los niveles de AF según años de escolaridad, fueron estimados mediante regresión lineal. El comando "svy" y "margins" fue utilizado para generar los promedios de AF ajustados por edad y sus respectivos 95\% IC.

La prevalencia de inactividad física ajustada por edad según años de escolaridad fue estimada mediante regresión logística para muestras complejas, estos resultados fueron presentados como prevalencia y sus respectivos $95 \%$ IC. El valor P para trend o tendencia fue estimado con regresión logística.

Para estimar la probabilidad de no cumplir con las recomendaciones de AF según años de escolaridad, se codificó AF como variable binaria donde personas físicamente activas fueron el grupo de referencia (valor asignado " 0 ") y las personas físicamente inactivas correspondieron al grupo de casos (valor asignado "1"). El grupo de referencia para años de escolaridad correspondió a 12 años, ya que el mayor porcentaje de la población se concentra en esta categoría (25,9\%). Posteriormente se procedió a realizar análisis de regresión logística no lineal entre AF y años de escolaridad codificados como variable continua (de 0 a 16 años). Los resultados fueron presentados como odds ratio (OR) ajustados por edad, con sus respectivos 95\% IC. Por tanto, un OR $>1$ indicó una mayor probabilidad de ser físicamente inactivo, mientras que un $\mathrm{OR}<1$ implicó una menor probabilidad. El nivel de significancia fue definido como $\mathrm{p}<0,05$.

\section{Resultados}

En la Tabla 1 se presentan las características de la población estudiada según años de escolari- dad. Un $12,6 \%$ y $10,6 \%$ de las mujeres y hombres reportaron no tener ningún año de escolaridad, mientras que la mayor proporción reporto tener entre 9-12 años de escolaridad (43,1\% y 44,4\%, para mujeres y hombres, respectivamente). También se observó que personas con un menor nivel de escolaridad presentaron una mayor edad en comparación a personas con una mayor escolaridad. Las diferencias de edad entre personas sin educación y aquellas con $>16$ años de escolaridad fue de aproximadamente 25 años. Diferencias similares fueron observadas para IMC y perímetro de cintura, donde mujeres con menor escolaridad presentaron un nivel de adiposidad más alto que aquellas con mayor escolaridad, estas diferencias no fueron observadas para hombres. Sin embargo, la prevalencia de obesidad determinada mediante IMC y obesidad central obtenida a través del perímetro de cintura fueron menores en personas con mayor escolaridad en comparación a personas clasificadas en la categoría sin educación (Tabla 1).

Con relación a la práctica de AF, la Figura 1 muestra el tiempo destinado a AF total, de transporte, AF moderada y AF vigorosa según años de escolaridad. En esta se observa que, en todos los niveles, los hombres presentaron mayores niveles de AF total, de transporte y de intensidades moderada y vigorosa, acentuándose estas diferencias en las categorías de AF vigorosa, MVPA y AF total. Los mayores niveles de AF moderada, vigorosa y total, en ambos sexos, se observaron en personas con 5 a 12 años de estudio, mientras que los niveles más bajos de AF se observaron en personas con $<4$ años de estudio. Cabe mencionar que, si bien los hombres reportaron un mayor nivel de AF de transporte a través de todas las categorías en comparación a las mujeres, los niveles de AF de transporte en ambos sexos fue similar para las diferentes categorías de nivel de escolaridad (Figura 1). El tiempo destinado a estar sentado aumentó en la medida que incrementaron los niveles de escolaridad, tanto en hombres como mujeres (Figura 1).

En la Figura 2 se presenta la prevalencia de inactividad física según años de escolaridad. Para ambos sexos se observó un descenso lineal de la prevalencia de inactividad física en la medida que incrementan los años de escolaridad. En mujeres, la prevalencia de inactividad física fluctuó entre $44,3 \%$ y $26,7 \%$ para personas sin educación versus aquellas con $>16$ años de escolaridad, respectiva- 
Tabla 1. Características sociodemográficas y nutricionales de la población según nivel educacional y sexo

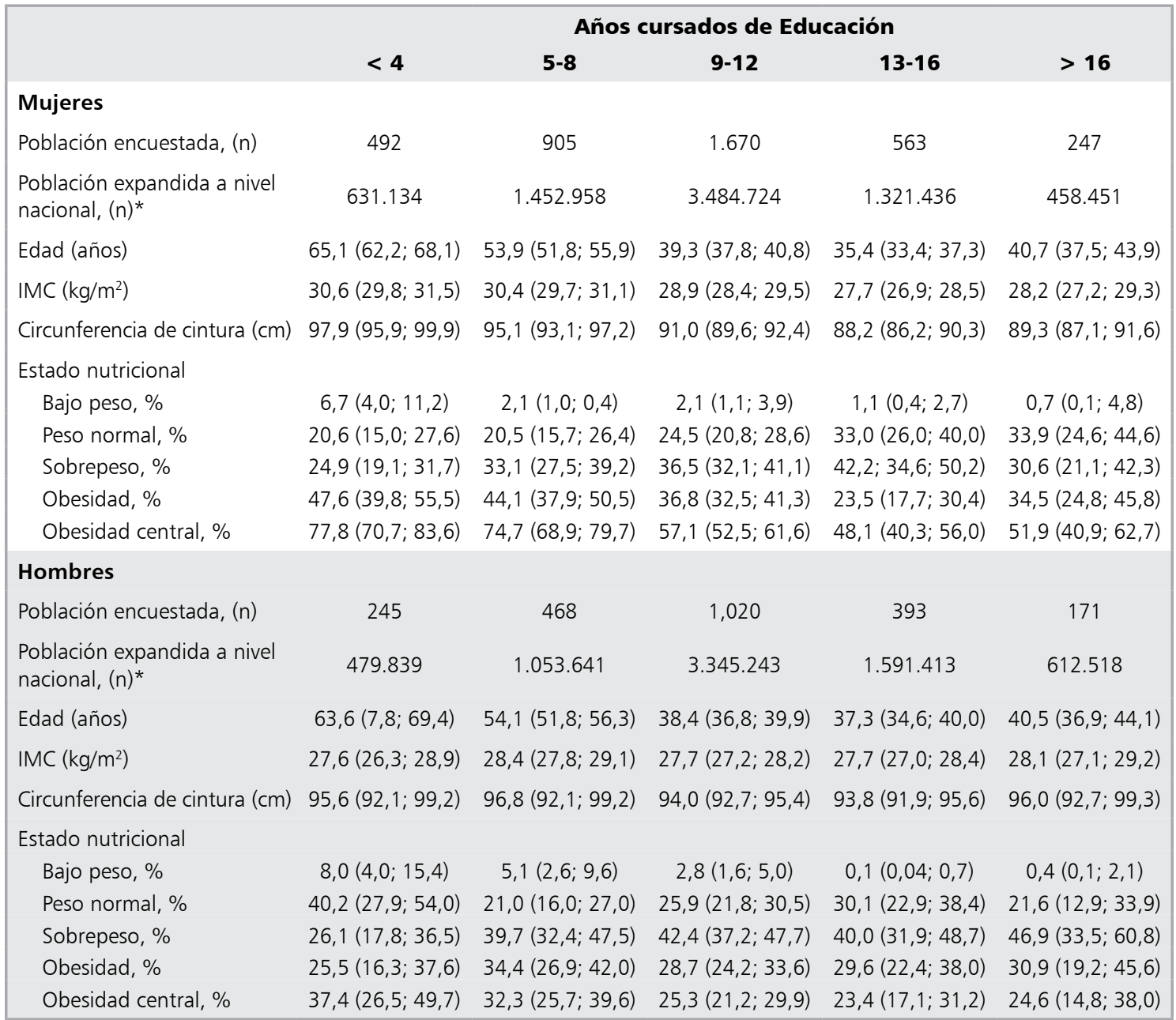

Datos son presentados como promedio para variables continuas y como prevalencia para variables categóricas y sus respectivos intervalos de confianza del 95\%. *Población expandida a nivel nacional estimada mediante el factor de ponderación de la ENS 2016-2017.

mente. En hombres, la prevalencia de inactividad física fue de $27,4 \%$ para la categoría sin educación y de un $14,7 \%$ para el grupo con $>16$ años de escolaridad (Figura 2).

La asociación entre años de escolaridad y la no adherencia a las recomendaciones de AF se presenta en la Figura 3. En comparación al grupo de referencia (personas con 12 años de escolaridad) mujeres y hombres con menos de 8 años de escolaridad presentaron una mayor probabilidad de no cumplir con las recomendaciones de actividad física, mientras que una escolaridad sobre 8 años no presentó diferencias significativas en comparación al grupo de referencia (12 años). Si bien la asociación entre inactividad física y educación mostró la misma dirección de asociación para hombres y mujeres, los hombres sin educación tenían una mayor probabilidad de ser físicamente inactivos en comparación a las mujeres en la misma categoría educacional (OR hombres: 3,97 [95\% IC: 2,27; 6,95], $\mathrm{p}<0,0001$; OR mujeres: 2,30 [95\% IC: 1,$57 ; 3,38$ ], $\mathrm{p}<0,0001)$. Sin embargo, estas diferencias fueron entre sexos a mayor nivel de escolaridad (Figura 3). 


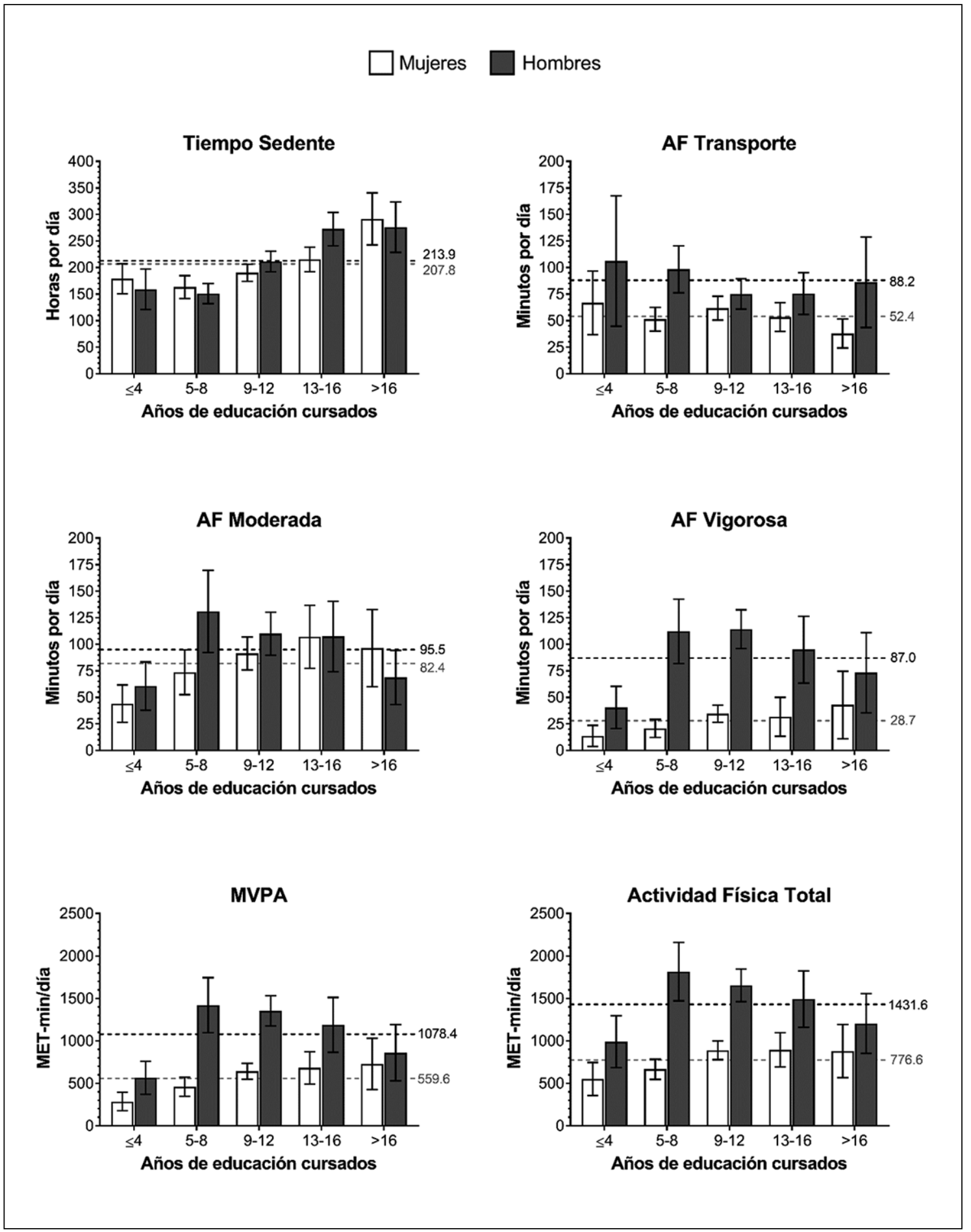

Figura 1. Niveles de actividad física y tiempo sedente según años de educación cursados. Datos presentados como medias y sus respectivos 95\% IC. Los análisis fueron ajustados por edad. La línea punteada de color gris y la línea punteada de color negro indica indican la media para cada uno de subcomponentes de actividad física para mujeres y hombres, respectivamente. 

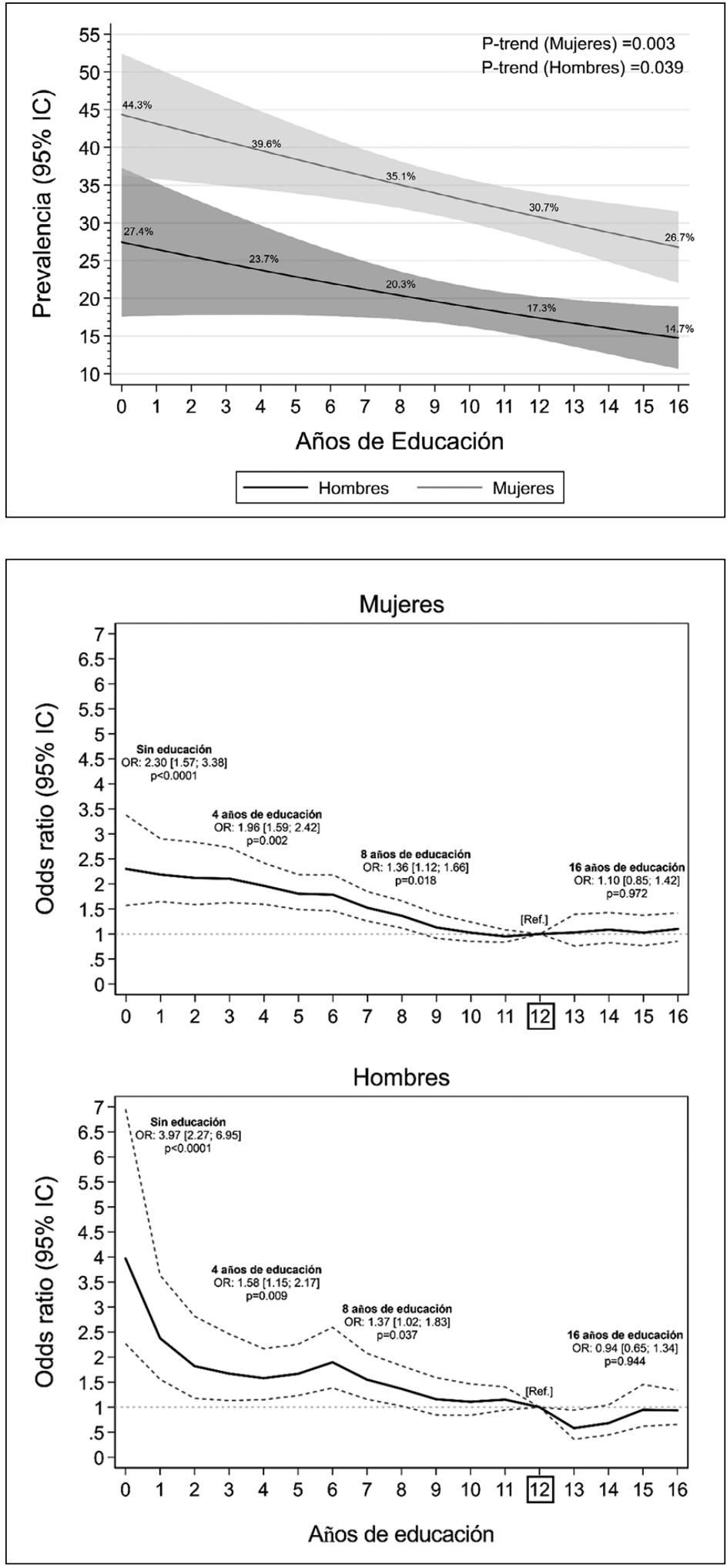

Figura 2. Prevalencia de inactividad física según años de educación cursados y sexo. Datos presentados como prevalencia ajustada por edad y sus respectivos 95\% IC. Los P-trend fueron estimados con regresión logística para muestras complejas e indican si hay una disminución significativa en la prevalencia de inactividad física al incrementar el nivel de escolaridad en mujeres y hombres.

Figura 3. Asociación entre nivel educacional y la no adherencia a las recomendaciones de actividad física. Datos presentados como odds ratio y sus respectivos $95 \%$ IC según sexo. Los análisis fueron ajustados por edad. El grupo de referencia corresponde a participantes que auto reportaron haber cursado 8 años de educación. Un valor de OR $>1$ indica una mayor probabilidad de ser físicamente inactivo, es decir, realizar menos de 150 minutos de AF de intensidad moderada o 75 minutos de AF vigorosa por semana. 


\section{Discusión}

\section{Principales resultados}

Los principales resultados de este estudio sugieren que, aquellos sectores de la población con menores niveles de estudio presentaron una mayor probabilidad de no cumplir con las recomendaciones de AF en comparación a aquellas personas con 12 años de estudio o más. Considerando los efectos nocivos para la salud asociados a la inactividad física ${ }^{1-6}$, la evidencia entregada por este estudio podría ser de utilidad para informar a las entidades gubernamentales respecto a que los años de estudio pueden marcar una tendencia o establecer una relación respecto al cumplimiento de las recomendaciones de AF, por lo tanto, se pueden implementar programas de promoción y mejoramiento de espacios públicos y privados para su práctica regular, especialmente enfocados a los sectores de la población más vulnerables a ser físicamente inactivos.

\section{Comparación con otros estudios}

Los principales hallazgos del estudio demuestran que existe una estrecha relación entre los años de estudio y la adherencia a realizar AF, en concordancia con lo reportado en otras poblaciones ${ }^{17-20}$. Bauman et al., reportaron en población asiática y australiana la existencia de una estrecha asociación entre el nivel educativo y la práctica de $\mathrm{AF}$, donde los grupos con mayores años de estudio fueron más activos, comparados con los de menor nivel educativo ${ }^{17}$. Gidlow et al., tras realizar una revisión sistemática de 28 estudios transversales y 5 longitudinales en 10 países, identificaron que en la mayoría de los estudios transversales existía una relación positiva entre nivel educativo y práctica de AF, es decir, a mayor educación, mayores son los niveles de AF reportados por la población ${ }^{21}$. De igual forma, un estudio realizado en 2.177 personas en Argentina (rango de edad de 15 a 64 años), reportó la existencia de asociación entre el nivel educativo y la AF ocupacional en hombres y mujeres. En este estudio, las personas con menos años de estudio reportaron mayor nivel de AF laboral, mientras que las personas con más años de estudio acumularon mayores niveles de AF en el tiempo de ocio ${ }^{22}$. Estos resultados concuerdan con una reciente revisión sistemática de la literatura que incluyó a 56 estudios de 30 países a nivel mundial, donde AF de tiempo libre o recreacional se asoció positivamente a nivel socio-económico, sin embargo, no se encontró relación entre nivel socio-económico y actividad física laboral ${ }^{23}$.

\section{Implicaciones de los resultados}

Un estudio reciente realizado en población británica, reportó que individuos con bajos niveles educativos o ingresos económicos presentan una alta prevalencia de estilos de vida no saludable en comparación a aquellos con niveles altos de estudio $^{12}$. El estudio estimó que el riesgo de mortalidad debido a enfermedades cardiovasculares fue 4,8 veces mayor (o su equivalente $480 \%$ ) en personas con un estilo de vida no saludable y con bajo nivel socioeconómico, mientras que personas con el mismo nivel socioeconómico, pero que presentaban una alta adherencia a estilos de vida saludable disminuyeron el riesgo de mortalidad cardiovascular a un $96 \%$. Resultados similares han sido reportados en población chilena, donde una mayor adherencia a estilos de vida saludable (caracterizados por cumplir con las recomendaciones internacionales para el consumo de alcohol, sal, frutas y verduras, actividad física, sedentarismo, horas de sueño y tabaquismo) se ha asociado a una menor probabilidad de desarrollar obesidad, diabetes mellitus tipo 2, hipertensión arterial y triglicéridos elevados ${ }^{24-26}$. Estos resultados destacan claramente la necesidad de mejorar la adherencia a la práctica regular de $\mathrm{AF}$, como también a otros estilos de vida saludable en estos sectores más vulnerables de la población.

\section{Fortalezas y limitaciones del estudio}

La principal fortaleza del estudio corresponde a que sus resultados son representativos para la población chilena e incluyen un alto espectro y detalle de los años de estudio y de práctica de AF de los participantes. A su vez, los datos relacionados con los niveles de AF han sido recolectados utilizando instrumentos validados ${ }^{14-16}$. Sin embargo, dentro de las limitantes de este estudio se encuentran la modalidad de auto-reporte para determinar la AF, lo cual presenta una baja correlación con métodos objetivos para la cuantificación de los niveles de $\mathrm{AF}$, como, por ejemplo, el uso de acelerómetros ${ }^{27}$. Por eso, las asociaciones observadas en este estudio podrían estar sesgadas por la sobreestimación de la AF auto reportada, principalmente en personas con estilos de vida no saludable, con patologías existentes y aquellas con sobrepeso u obesidad ${ }^{27}$. 
Otra limitación corresponde al uso de nivel de escolaridad como variable de exposición, la cual podría presentar asociaciones diferentes con AF en comparación a otros marcadores sociodemográficos como nivel de ingreso y nivel socioeconómico. Por último, es importante considerar que los resultados presentados en este estudio incluyen $\mathrm{AF}$ total, por ende, es relevante que estudios futuros verifiquen la asociación entre nivel de escolaridad y niveles de AF en un contexto laboral como de tiempo libre.

En conclusión, este estudio evidencia que sectores de la población con bajos niveles educativos son más proclives a no cumplir con las recomendaciones de $\mathrm{AF}$, en comparación a personas que reportaron tener 12 años de educación e incluso aquellas personas que reportaron haber cursado $>$ 16 años. Si consideramos que el no cumplir con las recomendaciones de AF se asocia a un incremento en el riesgo de desarrollar enfermedades cardiovasculares, cáncer y de morir prematuramente ${ }^{1-6}$, es importante generar evidencia que permita identificar a sectores de la población que son más vulnerables a ser físicamente inactivos, con el objetivo de poder implementar políticas públicas que promuevan la practica regular de actividad física.

Agradecimientos: Se agradece de manera especial a todos los participantes de la ENS 2016-17, al equipo profesional del Departamento de Salud Pública de la Facultad de Medicina de la Pontificia Universidad Católica de Chile, quienes desarrollaron y aplicaron la encuesta Nacional de Salud, y al Ministerio de Salud del Gobierno de Chile. Todos los autores revisaron críticamente el manuscrito y están de acuerdo con su versión final. Ninguno de los autores reporta tener conflictos de intereses.

\section{Referencias}

1. WHO. Global recommendations on physical activity for health. 2010. http://www.who.int/dietphysicalactivity/ publications/9789241599979/en/

2. WHO. The global action plan on physical activity 2018-2030. 2018. http://www.who.int/ncds/prevention/ physical-activity/gappa/action-plan

3. Celis-Morales CA, Lyall DM, Anderson J, Iliodromiti S, Fan Y, Ntuk UE, et al. The association between physical activity and risk of mortality is modulated by grip strength and cardiorespiratory fitness: evidence from
498,135 UK-Biobank participants. Eur Heart J 2016; 38 (2): 116-22.

4. Celis-Morales CA, Salas C, Álvarez C, Aguilar Farías N, Ramírez Campillos R, Leppe J, et al. Higher physical activity levels are associated with lower prevalence of cardiovascular risk factors in Chile. Rev Med Chile 2015; 143 (11): 1435-43.

5. Celis-Morales CA, Gray S, Petermann F, Iliodromiti S, Welsh P, Lyall DM, et al. Walking Pace Is Associated with Lower Risk of All-Cause and Cause-Specific Mortality. Med Sci Sports Exerc 2019; 51 (3): 472-80.

6. Celis-Morales CA, Lyall DM, Welsh P, Anderson J, Steell $\mathrm{L}$, Guo Y, et al. Association between active commuting and incident cardiovascular disease, cancer, and mortality: prospective cohort study. BMJ 2017; 357 (4): j1456.

7. Guthold R, Stevens GA, Riley LM, Bull F. Worldwide trends in insufficient physical activity from 2001 to 2016: a pooled analysis of 358 population-based surveys with 1.9 million participants. Lancet Glob Health 2018; 6 (10): e1077-e86.

8. MINSAL. Encuesta Nacional de Salud 2016-2017. Ministerio de Salud. 2017. http://www.ipsuss.cl/ipsuss/site/ artic/20171122/asocfile/20171122142253/ens_2016_17_ primeros_resultados.pdf.

9. Celis-Morales C, Salas C, Alduhishy A, Sanzana R, Martínez MA, Leiva A, et al. Socio-demographic patterns of physical activity and sedentary behaviour in Chile: results from the National Health Survey 2009-2010. J Public Health (Oxf) 2016; 38 (2): e98-e105.

10. Concha-Cisternas Y, Petermann-Rocha F, Garrido-Méndez A, Díaz-Martínez X, Leiva A, Salas-Bravo C, et al. Caracterización de los patrones de actividad física en distintos grupos etarios chilenos. Nutr Hosp 2019; 36 (1): 149-58.

11. Díaz-Martínez X, Garrido-Méndez A, Martínez M, Leiva A, Álvarez C, Ramírez-Campillo R, et al. Factores asociados a inactividad física en Chile: resultados de la Encuesta Nacional de Salud 2009-2010. Rev Med Chile 2017; 145 (10): 1259-67.

12. Foster HME, Celis-Morales CA, Nicholl BI, Petermann-Rocha F, Pell JP, Gill JMR, et al. The effect of socioeconomic deprivation on the association between an extended measurement of unhealthy lifestyle factors and health outcomes: a prospective analysis of the UK Biobank cohort. Lancet Public Health 2018; 3 (12): e576-e85.

13. WHO. Global Physical Activity Questionnaire: GPAQ version 2.0. 2009. http://www.who.int/chp/steps/resources/GPAQ_Analysis_Guide.pdf.

14. Aguilar-Farías N, Leppe J. Is a single question of the Global Physical Activity Questionnaire (GPAQ) valid 
for measuring sedentary behaviour in the Chilean population? J Sports Sci 2016; 35 (16): 1652-7.

15. Bull FC, Maslin TS, Armstrong T. Global Physical Activity Questionnaire (GPAQ): Nine Country Reliability and Validity Study. J Phys Act Health 2009; 6 (6): 790804.

16. Hoos T, Espinoza N, Marshall S, Arredondo EM. Validity of the Global Physical Activity Questionnaire (GPAQ) in Adult Latinas. J Phys Act Health 2012; 9 (5): 698-705.

17. Bauman A, Ma G, Cuevas F, Omar Z, Waqanivalu T, Phongsavan $\mathrm{P}$, et al. Cross-national comparisons of socioeconomic differences in the prevalence of leisure-time and occupational physical activity, and active commuting in six Asia-Pacific countries. J Epidemiol Community Health 2011; 65 (1): 35-43.

18. Bauman A, Ainsworth BE, Sallis JF, Hagströmer M, Craig CL, Bull FC, et al. The Descriptive Epidemiology of Sitting A 20-Country Comparison Using the International Physical Activity Questionnaire (IPAQ). Am J Prev Med 2011; 41 (2): 228-35.

19. Bauman A, Bull F, Chey T, Craig CL, Ainsworth BE, Sallis JF, et al. The International Prevalence Study on Physical Activity: results from 20 countries. Int J Behav Nutr Phys Act 2009; 6: 21.

20. Bauman AE, Reis RS, Sallis JF, Wells JC, Loos RJ, Martin BW. Correlates of physical activity: why are some people physically active and others not? Lancet 2012; 380 (9838): 258-71.

21. Gidlow C, Johnston LH, Crone D, Ellis N, James D. A. systematic review of the relationship between socio-eco- nomic position and physical activity. Health Educ J 2006; 65 (4): 338-67.

22. Tarducci G, Barengo N, Morea G, Gárgano S, Gandini A, Paganini A, et al. Relación entre el nivel de escolaridad y el patrón de actividad física en Balcarce, Argentina. Hacia promoc. Salud 2016; 21: 89-98.

23. Stalsberg R, Pedersen AV. Are Differences in Physical Activity across Socioeconomic Groups Associated with Choice of Physical Activity Variables to Report? Int J Environ Res Public Health 2018; 15 (5): 922.

24. Petermann-Rocha F, Durán E, Labraña AM, Martínez M, Leiva A, Garrido-Méndez A, et al. Factores asociados al desarrollo de obesidad en Chile: resultados de la Encuesta Nacional de Salud 2009-2010. Rev Med Chile 2017; 145: 716-22.

25. Petermann-Rocha F, Sillars A, Brown R, Sweeney L, Troncoso C, García-Hermoso A, et al. Sociodemographic patterns of urine sodium excretion and its association with hypertension in Chile: a cross-sectional analysis. Public Health Nutr 2019; 22 (11): 2012-21.

26. Leiva AM, Petermann-Rocha F, Martínez-Sanguinetti MA, Troncoso-Pantoja C, Concha Y, Garrido-Méndez A, et al. Asociación de un índice de estilos de vida saludable con factores de riesgo cardiovascular en población chilena. Rev Med Chile 2018; 146: 1405-14.

27. Celis-Morales CA, Pérez-Bravo F, Ibáñez L, Salas C, Bailey ME, Gill JM. Objective vs. self-reported physical activity and sedentary time: effects of measurement method on relationships with risk biomarkers. PLoS ONE 2012; 7 (5): e36345. 